Discrete Comput Geom 31:503-514 (2004)

DOI: $10.1007 / \mathrm{s} 00454-003-0818-3$

Geometry

\title{
Large Cells in Poisson-Delaunay Tessellations
}

\author{
Daniel Hug and Rolf Schneider \\ Mathematisches Institut, Albert-Ludwigs-Universität, \\ D-79104 Freiburg i. Br., Germany \\ \{daniel.hug,rolf.schneider\}@math.uni-freiburg.de
}

\begin{abstract}
It is proved that the shape of the typical cell of a Delaunay tessellation, derived from a stationary Poisson point process in $d$-dimensional Euclidean space, tends to the shape of a regular simplex, given that the volume of the typical cell tends to infinity. This follows from an estimate for the probability that the typical cell deviates by a given amount from regularity, given that its volume is large. As a tool for the proof, a stability result for simplices is established.
\end{abstract}

\section{Introduction and Main Result}

Voronoi tessellations (also called Voronoi mosaics or Voronoi diagrams) and their duals, Delaunay tessellations, are a thoroughly studied subject of discrete geometry. The book by Okabe et al. [11] gives an impression of the richness of the theory of these tessellations and of the variety of their applications. If the discrete point set in $\mathbb{R}^{d}$ from which such a tessellation is derived is random, one gets a random tessellation. Important examples are the Poisson-Voronoi and Poisson-Delaunay tessellations, which are derived from (stationary) Poisson point processes. We refer to Chapter 5 of [11], Chapter 10 of [14], and Chapter 6 of [13] for introductions to random tessellations.

A conjecture of D. G. Kendall initiated the study of limit shapes of large cells in special random mosaics. In the early 1940s Kendall conjectured (as documented in the introduction to the first edition of [14]) that the shape of the zero cell of the random tessellation generated by a stationary and isotropic Poisson line process in the plane tends to a circular shape given that the area of the zero cell tends to infinity. Contributions to this problem were made by Miles [9] and Goldman [1], and Kendall's conjecture was finally proved by Kovalenko [3], [5]. In [2] the limit shape for zero cells and typical cells of not necessarily isotropic, stationary Poisson hyperplane tessellations was found, and the probability of large deviations from the limit shape was estimated. Kovalenko [4] treated an analogue of Kendall's problem for the typical cell of a stationary Poisson- 
Voronoi tessellation in the plane. Again, the shape tends to circularity, given that the area tends to infinity.

The present paper is in a similar spirit. We consider the typical cell of a stationary Poisson-Delaunay mosaic in $d$-dimensional space and prove, as a consequence of a precise estimate, that its shape tends to that of a regular simplex, given that the volume tends to infinity.

Let $\tilde{X}$ be a stationary Poisson point process with intensity $\lambda>0$ in $d$-dimensional Euclidean space $\mathbb{R}^{d}(d \geq 2)$. Let $Y$ denote the Poisson-Delaunay tessellation derived from $\tilde{X}$. The typical cell of $Y$ (as defined in Section 6.2 of [13]) is denoted by $Z$ (explanations are given below in Section 2). Almost surely, $Z$ is a simplex which is inscribed to a sphere centered at the origin.

For the formulation of our result, we need a measure for the deviation of the shape of a simplex from the shape of a regular simplex. For $d$-simplices $S_{1}, S_{2}$, we define $\eta\left(S_{1}, S_{2}\right)$ as the smallest number $\eta$ with the property that for each vertex $p$ of one of the simplices there is a vertex $q$ of the other such that $\|p-q\| \leq \eta$ (here $\|\cdot\|$ denotes the Euclidean norm). Note that $\delta\left(S_{1}, S_{2}\right) \leq \eta\left(S_{1}, S_{2}\right)$, where $\delta$ denotes the Hausdorff metric (see Section 1.8 of [12]). For a $d$-simplex $S$, let $z$ be the center and let $r$ be the radius of the sphere through the vertices of $S$, and set

$$
\rho(S):=\min \left\{\eta\left(r^{-1}(S-z), T\right): T \in \mathcal{T}^{d}\right\},
$$

where $\mathcal{T}^{d}$ denotes the set of regular simplices inscribed to the unit sphere $S^{d-1}$.

By $\mathbb{P}$ we denote the underlying probability, and $\mathbb{P}(\cdot \mid \cdot)$ is a conditional probability. We write $V_{d}$ for the volume in $\mathbb{R}^{d}$.

Theorem 1. Let $Y$ denote the Poisson-Delaunay tessellation derived from a stationary Poisson process with intensity $\lambda>0$ in $\mathbb{R}^{d}$; let $Z$ be its typical cell. There is a constant $c_{0}$ depending only on $d$ such that the following is true. If $\varepsilon \in(0,1)$ and $I=[a, b)$ is any interval (possibly $b=\infty$ ) with $a \lambda \geq \sigma_{0}>0$, then

$$
\mathbb{P}\left(\rho(Z) \geq \varepsilon \mid V_{d}(Z) \in I\right) \leq c \exp \left\{-c_{0} \varepsilon^{2} a \lambda\right\},
$$

where $c$ is a constant depending only on $d, \varepsilon$, and $\sigma_{0}$.

As a consequence, we have

$$
\lim _{a \rightarrow \infty} \mathbb{P}\left(\rho(Z) \geq \varepsilon \mid V_{d}(Z) \geq a\right)=0
$$

for any fixed $\varepsilon>0$.

Using similar arguments as in [2], one can also deduce a corresponding result for the zero cell $Z_{0}$ (the cell containing the origin of $\mathbb{R}^{d}$ ) of $Y$. This will not be carried out here, since the procedure is clear from [2].

The proof of Theorem 1 is based on a geometric stability result for simplices (Theorem 2 in Section 3) and on two estimates, provided by Lemmas 2 and 3 in Section 4. The derivation of these estimates is facilitated by an explicit formula for the distribution of $Z$, which is due to R. E. Miles. 


\section{The Typical Cell of a Poisson-Delaunay Tessellation}

We recall briefly the notion of a Poisson-Delaunay tessellation and its typical cell (details can be found, e.g., in [13]). Let $\tilde{X}$ be a stationary Poisson process in $\mathbb{R}^{d}$, with intensity $\lambda>0$. With probability one, no $d+1$ points of $\tilde{X}$ are in a hyperplane, and no $d+2$ points lie on a sphere. If $d+1$ points $x_{1}, \ldots, x_{d+1}$ of $\tilde{X}$ lie on a sphere that contains no point of $\tilde{X}$ in its interior, then the convex hull of $x_{1}, \ldots, x_{d+1}$ is called a cell. The set $Y$ of all such cells is a tessellation of $\mathbb{R}^{d}$ by simplices, the Poisson-Delaunay tessellation derived from $\tilde{X}$. (This construction of a Delaunay tessellation is equivalent to the usual one as the dual of a Voronoi tessellation.) Since $Y$ can be considered as a stationary particle process (of intensity $\lambda^{\prime}=[(d+1) a(d)]^{-1} \lambda$, where $a(d)$ is given by (1) below), one can associate with it a shape distribution (see Section 4.2 of [13]). It can be described as follows. For a $d$-simplex $S$, we denote by $z(S)$ the center of the sphere through the vertices of $S$. Let $\Delta_{0}$ be the set of all $d$-simplices $S$ in $\mathbb{R}^{d}$ with $z(S)=0$. Let $C^{d}$ be the cube $\left[-\frac{1}{2}, \frac{1}{2}\right]^{d}$. The shape distribution of $Y$ is the probability measure $\mathbb{Q}_{0}$ on $\Delta_{0}$ with the property that

$$
\mathbb{Q}_{0}(\mathcal{A})=\left(1 / \lambda^{\prime}\right) \mathbb{E} \operatorname{card}\left\{S \in Y: z(S) \in C^{d}, S-z(S) \in \mathcal{A}\right\}
$$

for Borel sets $\mathcal{A} \subset \Delta_{0}$; here $\mathbb{E}$ denotes mathematical expectation. The typical cell of the Poisson-Delaunay tessellation $Y$ is defined as a random polytope with distribution $\mathbb{Q}_{0}$. A more intuitive interpretation of this distribution is possible due to the fact that stationary Poisson-Delaunay tessellations are mixing and hence ergodic [13, Satz 6.4.2]. This entails that, for $\mathcal{A}$ as before,

$$
\mathbb{Q}_{0}(\mathcal{A})=\lim _{r \rightarrow \infty} \frac{\operatorname{card}\left\{S \in Y: z(S) \in r C^{d}, S-z(S) \in \mathcal{A}\right\}}{\operatorname{card}\left\{S \in Y: z(S) \in r C^{d}\right\}}
$$

holds with probability one.

For example, suppose we are interested in $\mathbb{P}\left(V_{d}(Z) \geq a\right)$, the probability that the typical cell has volume at least $a>0$. Then we can take an arbitrary realization of the tessellation $Y$ and a large number $r$ and consider, among the cells $S$ of the realization with center $z(S)$ in the cube $r C^{d}$, the relative frequency of those with volume at least $a$. This proportion will almost surely be a good approximation to the probability $\mathbb{P}\left(V_{d}(Z) \geq a\right)$.

We make use of the explicit integral representation of the distribution $\mathbb{Q}_{0}$ given by Lemma 1. It is due to Miles [8, formula (76)]; the proof can also be found in [10, Theorem 7.5] and [13, Satz 6.2.10]. Let $\sigma$ denote the spherical Lebesgue measure on the unit sphere $S^{d-1}$, and let $\kappa_{d}$ be the volume of the $d$-dimensional unit ball.

Lemma 1. Let $Y$ be the Delaunay tessellation derived from a stationary Poisson process of intensity $\lambda>0$ in $\mathbb{R}^{d}$, and let $\mathbb{Q}_{0}$ be the distribution of its typical cell. Let $\mathcal{A} \subset \Delta_{0}$ be a Borel set. Then

$$
\begin{aligned}
\mathbb{Q}_{0}(\mathcal{A})= & a(d) \lambda^{d} \int_{0}^{\infty} \int_{S^{d-1}} \ldots \int_{S^{d-1}} \mathbf{1}_{\mathcal{A}}\left(\operatorname{conv}\left\{r u_{0}, \ldots, r u_{d}\right\}\right) e^{-\lambda \kappa_{d} r^{d}} r^{d^{2}-1} \\
& \times V_{d}\left(\operatorname{conv}\left\{u_{0}, \ldots, u_{d}\right\}\right) d \sigma\left(u_{0}\right) \cdots d \sigma\left(u_{d}\right) d r
\end{aligned}
$$


with

$$
a(d):=\frac{d^{2}}{2^{d+1} \pi^{(d-1) / 2}} \frac{\Gamma\left(d^{2} / 2\right)}{\Gamma\left(\left(d^{2}+1\right) / 2\right)}\left[\frac{\Gamma((d+1) / 2)}{\Gamma(d / 2+1)}\right]^{d} .
$$

\section{A Stability Result for Simplices}

For a $d$-dimensional simplex $S \subset \mathbb{R}^{d}$ we say that $S$ is inscribed to the unit sphere $S^{d-1}$ if the vertices of $S$ lie on $S^{d-1}$. Let $S$ be such a simplex and suppose that it has maximal volume among all simplices inscribed to $S^{d-1}$. Then it is easy to see that $S$ is a regular simplex (e.g., p. 317 of [7]). Here we need an improved version of such a 'uniqueness' result, in the form of a stability estimate.

In the following, $T^{d}$ is a regular simplex inscribed to $S^{d-1}$.

Theorem 2. There is a positive constant $c(d)$ such that the following is true for any $\varepsilon \in[0,1]$. If $S$ is a simplex inscribed to $S^{d-1}$ and if $\rho(S) \geq \varepsilon$, then

$$
V_{d}(S) \leq\left(1-c(d) \varepsilon^{2}\right) V_{d}\left(T^{d}\right) .
$$

Proof. First we consider the case $d=2$, where we show that $c(2)=\frac{1}{12}$ is a possible choice.

Let $S$ be a triangle inscribed to $S^{1}$ and satisfying $V_{2}(S)>\left(1-\varepsilon^{2} / 12\right) V_{2}\left(T^{2}\right)$; here $V_{2}\left(T^{2}\right)=3 \sqrt{3} / 4$. Then $0 \in$ int $S$. Let $2 \alpha, 2 \beta$, and $2 \gamma$ be the angles at 0 spanned by the edges of $S$. Then

$$
V_{2}(S)=\sin \alpha \cos \alpha+\sin \beta \cos \beta+\sin \gamma \cos \gamma
$$

and $\alpha+\beta+\gamma=\pi$. We can choose the notation in such a way that the angles $\varphi:=\alpha-\pi / 3$ and $\psi:=\beta-\pi / 3$ are either both non-negative or both non-positive. An elementary calculation gives

$$
V_{2}(S)-V_{2}\left(T^{2}\right)=(\sqrt{3} / 2)\left[\cos ^{2} \varphi+\cos ^{2} \psi-2\right]-\sin (\varphi+\psi)[\sin \varphi \sin \beta+\sin \psi \sin \alpha] .
$$

Since either $\varphi \geq 0, \psi \geq 0$ or $\varphi \leq 0, \psi \leq 0$ (and $|\varphi+\psi|<\pi$ ), we get

$$
\sin (\varphi+\psi)[\sin \varphi \sin \beta+\sin \psi \sin \alpha] \geq 0 .
$$

We deduce that

$$
-\frac{\varepsilon^{2}}{12} \frac{3 \sqrt{3}}{4}<V_{2}(S)-V_{2}\left(T^{2}\right) \leq \frac{\sqrt{3}}{2}\left(\cos ^{2} \varphi-1\right),
$$

hence, observing that $-\pi / 3 \leq \varphi \leq \pi / 6$,

$$
1-\frac{1}{2} \varphi^{2} \geq \cos ^{2} \varphi>1-\frac{1}{8} \varepsilon^{2} .
$$

Thus $|\varphi|<\varepsilon / 2$, and similarly $|\psi|<\varepsilon / 2$, hence $|2 \alpha-2 \pi / 3|<\varepsilon$ and $|2 \beta-2 \pi / 3|<\varepsilon$. 
Let $p$ be the vertex of $S$ common to the edges spanning the angles $2 \alpha$ and $2 \beta$. Let $T$ be the regular triangle inscribed to $S^{1}$ with one vertex at $p$. Then $\eta(S, T)<\varepsilon$. This proves the assertion for $d=2$.

Now let $d \geq 3$, and assume that the assertion has been proved in dimension $d-1$.

Let $S$ be a $d$-simplex inscribed to $S^{d-1}$ and satisfying

$$
V_{d}(S)>(1-\alpha) V_{d}\left(T^{d}\right)
$$

for some given number $\alpha>0$. First we assume that $0 \in$ int $S$. The inradius $r(S)$ of $S$ satisfies $r(S) \leq 1 / d$ (see, e.g., Satz 1 of [6], or Lemma 13.2.2 of [7]). Hence, there is at least one facet of $S$, say $F$, which has a distance at most $1 / d$ from 0 . Therefore, there is a vector $u \in S^{d-1}$ and a number $t \in(0,1 / d]$ such that aff $F=H(u, t):=$ $\left\{z \in \mathbb{R}^{d}:\langle u, z\rangle=t\right\}$. Let $p$ be the vertex of $S$ not in $F$, and let $q$ be the point in $S^{d-1}$ with maximal distance from $H(u, t)$. Let $a$ be the distance between the hyperplanes through $p$ and $q$ parallel to $H(u, t)$. The $(d-1)$-volume of $F$ is less than or equal to the $(d-1)$-volume of a regular $(d-1)$-simplex inscribed to $H(u, t) \cap S^{d-1}$. Moreover, the function $x \mapsto\left(1-x^{2}\right)^{(d-1) / 2}(1+x)$ attains a unique maximum on the interval $[0,1]$ at $1 / d$. All this implies

$$
\begin{aligned}
V_{d}(S) & =\frac{1}{d} V_{d-1}(F)(1+t-a) \\
& \leq \frac{1}{d} V_{d-1}\left(T^{d-1}\right)\left(1-t^{2}\right)^{(d-1) / 2}(1+t-a) \\
& \leq \frac{1}{d} V_{d-1}\left(T^{d-1}\right)\left(1-t^{2}\right)^{(d-1) / 2}(1+t) \\
& \leq \frac{1}{d} V_{d-1}\left(T^{d-1}\right)\left(1-\frac{1}{d^{2}}\right)^{(d-1) / 2}\left(1+\frac{1}{d}\right) \\
& =V_{d}\left(T^{d}\right) \\
& <V_{d}(S)+\alpha V_{d}\left(T^{d}\right) .
\end{aligned}
$$

From this chain of inequalities, we draw three conclusions. In this proof, $c_{1}, c_{2}, \ldots$ denote positive constants depending only on the dimension $d$.

The first conclusion is that

$$
(1 / d) V_{d-1}\left(T^{d-1}\right)\left(1-t^{2}\right)^{(d-1) / 2} a<\alpha V_{d}\left(T^{d}\right) .
$$

Here $t \leq 1 / d$, hence $a<c_{1} \alpha$. Since $\|p-q\|^{2}=2 a$, we get

$$
\|p-q\|<c_{2} \sqrt{\alpha} .
$$

The second conclusion is that

$$
\frac{1}{d} V_{d-1}\left(T^{d-1}\right)\left[\left(1-\frac{1}{d^{2}}\right)^{(d-1) / 2}\left(1+\frac{1}{d}\right)-\left(1-t^{2}\right)^{(d-1) / 2}(1+t)\right]<\alpha V_{d}\left(T^{d}\right)
$$


Let

$$
g(x):=\left(1-x^{2}\right)^{(d-1) / 2}(1+x) \quad \text { for } \quad x \in[0,1] .
$$

The first two derivatives are given by

$$
g^{\prime}(x)=-\left(1-x^{2}\right)^{(d-3) / 2}\left(d x^{2}+(d-1) x-1\right)
$$

and

$$
g^{\prime \prime}(x)=(d-1)\left(1-x^{2}\right)^{(d-5) / 2}\left(d x^{3}+(d-2) x^{2}-3 x-1\right),
$$

for $x \in[0,1)$. Since $g^{\prime}(1 / d)=0$, we get

$$
g(x)=g\left(\frac{1}{d}\right)+\frac{1}{2} g^{\prime \prime}(\xi)\left(x-\frac{1}{d}\right)^{2}, \quad x \in\left[0, \frac{1}{d}\right],
$$

with a suitable $\xi \in[x, 1 / d]$. We estimate $g^{\prime \prime}$ from above by a negative constant. For this, we set

$$
f(x):=d x^{3}+(d-2) x^{2}-3 x-1 .
$$

An elementary discussion shows that $f$ is strictly decreasing in $[0,1 / d]$. In particular, we deduce that

$$
f(x) \leq f(0)=-1 \quad \text { for } \quad x \in[0,1 / d] .
$$

This shows that, for $x \in[0,1 / d]$,

$$
g^{\prime \prime}(x) \leq-(d-1)\left(1-x^{2}\right)^{(d-5) / 2} \leq \begin{cases}-(d-1), & d \in\{3,4\}, \\ -(d-1)\left(1-d^{-2}\right)^{(d-5) / 2}, & d \geq 5,\end{cases}
$$

hence

$$
\frac{1}{2} g^{\prime \prime}(x) \leq-c_{3} \quad \text { for } \quad x \in[0,1 / d]
$$

with $c_{3}>0$. Now (5) gives

$$
c_{3}(t-1 / d)^{2} \leq g(1 / d)-g(t),
$$

and from (4) we conclude that

$$
|t-1 / d|<c_{4} \sqrt{\alpha}
$$

Our third conclusion is that

$$
(1 / d)\left[V_{d-1}\left(T^{d-1}\right)\left(1-t^{2}\right)^{(d-1) / 2}-V_{d-1}(F)\right](1+t-a)<\alpha V_{d}\left(T^{d}\right) .
$$

Here $1+t-a>1+1 / d-c_{4} \sqrt{\alpha}-c_{1} \alpha>1$, if we assume that

$$
c_{4} \sqrt{\alpha}+c_{1} \alpha<1 / d
$$


The $(d-1)$-simplex $F^{\prime}:=\left(1-t^{2}\right)^{-1 / 2}(F-t u)$ is inscribed to $H(u, 0) \cap S^{d-1}$ and, as a consequence of (7) and of $t \leq 1 / d$, satisfies

$$
\begin{aligned}
V_{d-1}\left(F^{\prime}\right) & >V_{d-1}\left(T^{d-1}\right)-\alpha d V_{d}\left(T^{d}\right)\left(1-d^{-2}\right)^{(1-d) / 2} \\
& =\left(1-c_{5} \alpha\right) V_{d-1}\left(T^{d-1}\right)
\end{aligned}
$$

Let $c(d-1)$ be the constant appearing in the induction hypothesis. We assume that

$$
c_{5} \alpha / c(d-1) \leq 1
$$

and put $c_{5} \alpha / c(d-1)=: \gamma$. Then (9) and the induction hypothesis imply that $\eta\left(T^{d-1}, F^{\prime}\right)$ $<\sqrt{\gamma}$ for a suitably chosen regular $(d-1)$-dimensional simplex $T^{d-1} \subset H(u, 0) \cap S^{d-1}$. Let

$$
T:=\left(1-1 / d^{2}\right)^{1 / 2} T^{d-1}+\frac{1}{d} u
$$

and $T^{d}:=\operatorname{conv}(T \cup\{q\})$. Then $T^{d}$ is a regular $d$-simplex, inscribed to $S^{d-1}$. The two $(d-1)$-simplices $F$ and $T$ have the property that to each vertex $v$ of one of them there is a vertex $w$ of the other such that

$$
\begin{aligned}
\|v-w\| & <\sqrt{\left(1-t^{2}\right) \gamma}+\frac{1}{\sqrt{1-1 / d^{2}}}\left|t-\frac{1}{d}\right| \\
& \leq \sqrt{c_{5} \alpha / c(d-1)}+c_{6} \sqrt{\alpha} \leq c_{7} \sqrt{\alpha}
\end{aligned}
$$

by (6). Together with (3), this shows that

$$
\eta\left(S, T^{d}\right)<c_{8} \sqrt{\alpha}
$$

Now we choose $c(d)>0$ so small that $c_{8} \sqrt{c(d)} \leq 1$ and that $\alpha \leq c(d)$ implies (8) and (10).

Let $\varepsilon \in(0,1]$ be given, and put $\alpha:=c(d) \varepsilon^{2}$. Then

$$
V_{d}(S)>\left(1-c(d) \varepsilon^{2}\right) V_{d}\left(T^{d}\right)
$$

implies

$$
\eta\left(S, T^{d}\right)<\varepsilon .
$$

Finally, we can decrease $c(d)$, if necessary, so that $0 \notin$ int $S$ implies

$$
V_{d}(S) \leq(1-c(d)) V_{d}\left(T^{d}\right) .
$$

The induction step is now finished, hence the proof of Theorem 2 is complete.

Remark. The estimate (2) is of optimal order, that is, $\varepsilon^{2}$ cannot be replaced by a smaller power of $\varepsilon$. This is easily seen by appropriately moving one vertex of a regular simplex. 


\section{Proof of Theorem 1}

In the following, we set $\tau_{d}:=V_{d}\left(T^{d}\right)$. By $c_{1}, \ldots, c_{5}$ we denote positive constants depending only on $d$ or on $d$ and $\varepsilon$, as indicated. We write $c_{1}=c_{1}(d)$ for the constant $c(d)$ in Theorem 2 and $c_{2}=c_{2}(d)$ for the constant $a(d)$ of Lemma 1 in Section 2.

Lemma 2. For each $\varepsilon \in(0,1)$, there is a constant $c_{3}=c_{3}(d, \varepsilon)$ such that, for $0<$ $h \leq h_{0}:=\left(c_{1} /\left(c_{1}+12\right)\right) \varepsilon^{2}$ and $a \lambda>0$,

$$
\mathbb{P}\left(V_{d}(Z) \in a[1,1+h]\right) \geq c_{3} h(a \lambda)^{d} \exp \left\{-\left(\kappa_{d} / \tau_{d}\right)\left(1+\left(c_{1} / 4\right) \varepsilon^{2}\right) a \lambda\right\} .
$$

Proof. Let $\varepsilon \in(0,1), h \in\left(0, h_{0}\right]$ and $a>0, \lambda>0$ be given. For $x_{0}, \ldots, x_{d} \in \mathbb{R}^{d}$, we set $V_{d}\left(x_{0}, \ldots, x_{d}\right):=V_{d}\left(\operatorname{conv}\left\{x_{0}, \ldots, x_{d}\right\}\right)$. From Lemma 1 , we obtain

$$
\begin{aligned}
\mathbb{P}\left(V_{d}(Z) \in\right. & a[1,1+h]) \\
= & c_{2} \lambda^{d} \int_{0}^{\infty} \int_{S^{d-1}} \cdots \int_{S^{d-1}} \mathbf{1}\left\{r^{d} V_{d}\left(u_{0}, \ldots, u_{d}\right) \in a[1,1+h]\right\} \\
& \times \exp \left\{-\lambda \kappa_{d} r^{d}\right\} r^{d^{2}-1} V_{d}\left(u_{0}, \ldots, u_{d}\right) d \sigma\left(u_{0}\right) \cdots d \sigma\left(u_{d}\right) d r .
\end{aligned}
$$

Substituting $s=\lambda \kappa_{d} r^{d}$, we get

$$
\begin{aligned}
\mathbb{P}\left(V_{d}(Z) \in\right. & a[1,1+h]) \\
= & \frac{c_{2}}{d \kappa_{d}^{d}} \\
& \times \int_{S^{d-1}} \cdots \int_{S^{d-1}} \int_{0}^{\infty} \mathbf{1}\left\{s \in a \lambda \kappa_{d} / V_{d}\left(u_{0}, \ldots, u_{d}\right)[1,1+h]\right\} \\
& \times e^{-s} s^{d-1} d s V_{d}\left(u_{0}, \ldots, u_{d}\right) d \sigma\left(u_{0}\right) \cdots d \sigma\left(u_{d}\right) .
\end{aligned}
$$

For fixed $u_{0}, \ldots, u_{d} \in S^{d-1}$ in general position, we apply to the inner integral the mean value theorem for integrals. This gives the existence of some

$$
\xi\left(u_{0}, \ldots, u_{d}\right) \in a \lambda \kappa_{d} / V_{d}\left(u_{0}, \ldots, u_{d}\right)[1,1+h]
$$

such that

$$
\begin{aligned}
\mathbb{P}\left(V_{d}(Z) \in\right. & a[1,1+h]) \\
= & \frac{c_{2}}{d \kappa_{d}^{d-1}} h a \lambda \int_{S^{d-1}} \cdots \int_{S^{d-1}} \exp \left\{-\xi\left(u_{0}, \ldots, u_{d}\right)\right\} \\
& \times \xi\left(u_{0}, \ldots, u_{d}\right)^{d-1} d \sigma\left(u_{0}\right) \cdots d \sigma\left(u_{d}\right) \\
\geq & \frac{c_{2}}{d \kappa_{d}^{d-1}} h a \lambda \underbrace{\int \cdots \int}_{R(d, \varepsilon)} \exp \left\{-\xi\left(u_{0}, \ldots, u_{d}\right)\right\} \\
& \times \xi\left(u_{0}, \ldots, u_{d}\right)^{d-1} d \sigma\left(u_{0}\right) \cdots d \sigma\left(u_{d}\right),
\end{aligned}
$$


where

$$
R(d, \varepsilon):=\left\{\left(u_{0}, \ldots, u_{d}\right) \in\left(S^{d-1}\right)^{d+1}: V_{d}\left(u_{0}, \ldots, u_{d}\right) \geq\left(1+\left(c_{1} / 12\right) \varepsilon^{2}\right)^{-1} \tau_{d}\right\} .
$$

For $\left(u_{0}, \ldots, u_{d}\right) \in R(d, \varepsilon)$ we can estimate

$$
\xi\left(u_{0}, \ldots, u_{d}\right) \geq a \lambda \kappa_{d} / \tau_{d}
$$

and

$$
\xi\left(u_{0}, \ldots, u_{d}\right) \leq\left(1+h_{0}\right)\left(1+\left(c_{1} / 12\right) \varepsilon^{2}\right) a \lambda \kappa_{d} / \tau_{d} .
$$

Since $\sigma^{d+1}(R(d, \varepsilon))$ depends only on $d$ and $\varepsilon$, this gives

$$
\begin{aligned}
\mathbb{P}\left(V_{d}(Z)\right. & \in a[1,1+h]) \\
& \geq c_{3}(d, \varepsilon) h(a \lambda)^{d} \exp \left\{-\left(\kappa_{d} / \tau_{d}\right)\left(1+h_{0}\right)\left(1+\left(c_{1} / 12\right) \varepsilon^{2}\right) a \lambda\right\} .
\end{aligned}
$$

Now

$$
\left(1+h_{0}\right)\left(1+\left(c_{1} / 12\right) \varepsilon^{2}\right) \leq 1+\left(c_{1} / 4\right) \varepsilon^{2},
$$

hence the assertion follows.

Lemma 3. For each $\varepsilon \in(0,1)$, there is a constant $c_{5}=c_{5}(d, \varepsilon)$ such that, for $a \lambda>0$ and $h>0$,

$$
\mathbb{P}\left(V_{d}(Z) \in a[1,1+h], \rho(Z) \geq \varepsilon\right) \leq c_{5} h a \lambda \exp \left\{-\left(\kappa_{d} / \tau_{d}\right)\left(1+\left(c_{1} / 2\right) \varepsilon^{2}\right) a \lambda\right\} .
$$

Proof. As in the proof of Lemma 2, we get

$$
\begin{aligned}
\mathbb{P}\left(V_{d}(Z) \in\right. & a[1,1+h], \rho(Z) \geq \varepsilon) \\
= & c_{2} \lambda^{d} \int_{0}^{\infty} \int_{S^{d-1}} \cdots \int_{S^{d-1}} \mathbf{1}\left\{r^{d} V_{d}\left(u_{0}, \ldots, u_{d}\right) \in a[1,1+h]\right\} \\
& \times \mathbf{1}\left\{\rho\left(\operatorname{conv}\left\{u_{0}, \ldots, u_{d}\right\}\right) \geq \varepsilon\right\} \\
& \times \exp \left\{-\lambda \kappa_{d} r^{d}\right\} r^{d^{2}-1} V_{d}\left(u_{0}, \ldots, u_{d}\right) d \sigma\left(u_{0}\right) \cdots d \sigma\left(u_{d}\right) d r \\
= & \frac{c_{2}}{d \kappa_{d}^{d}} \\
& \times \int_{S^{d-1}} \ldots \int_{S^{d-1}} \int_{0}^{\infty} \mathbf{1}\left\{s \in a \lambda \kappa_{d} / V_{d}\left(u_{0}, \ldots, u_{d}\right)[1,1+h]\right\} e^{-s} s^{d-1} d s \\
& \times \mathbf{1}\left\{\rho\left(\operatorname{conv}\left\{u_{0}, \ldots, u_{d}\right\}\right) \geq \varepsilon\right\} V_{d}\left(u_{0}, \ldots, u_{d}\right) d \sigma\left(u_{0}\right) \cdots d \sigma\left(u_{d}\right) \\
= & \frac{c_{2}}{d \kappa_{d}^{d-1}} h a \lambda \int_{S^{d-1}} \ldots \int_{S^{d-1}} \exp \left\{-\xi\left(u_{0}, \ldots, u_{d}\right)\right\} \xi\left(u_{0}, \ldots, u_{d}\right)^{d-1} \\
& \times \mathbf{1}\left\{\rho\left(\operatorname{conv}\left\{u_{0}, \ldots, u_{d}\right\}\right) \geq \varepsilon\right\} d \sigma\left(u_{0}\right) \cdots d \sigma\left(u_{d}\right),
\end{aligned}
$$

where again $(12)$ holds. By Theorem 2 , the inequality $\rho\left(\operatorname{conv}\left\{u_{0}, \ldots, u_{d}\right\}\right) \geq \varepsilon$ implies

$$
V_{d}\left(u_{0}, \ldots, u_{d}\right) \leq\left(1-c_{1} \varepsilon^{2}\right) \tau_{d} .
$$


Hence, if $\mathbf{1}\left\{\rho\left(\operatorname{conv}\left\{u_{0}, \ldots, u_{d}\right\}\right) \geq \varepsilon\right\} \neq 0$, then

$$
\xi\left(u_{0}, \ldots, u_{d}\right) \geq \kappa_{d} a \lambda\left(1-c_{1} \varepsilon^{2}\right)^{-1} \tau_{d}^{-1} \geq\left(\kappa_{d} / \tau_{d}\right)\left(1+c_{1} \varepsilon^{2}\right) a \lambda .
$$

Moreover, there is a constant $c_{4}=c_{4}(d, \varepsilon)$ such that, for $\xi \geq 0$,

$$
\exp (-\xi) \xi^{d-1} \leq c_{4} \exp \left\{-\left(1-\frac{c_{1}}{2\left(1+c_{1}\right)} \varepsilon^{2}\right) \xi\right\}
$$

The estimates (13) and (14) imply, for $\xi=\xi\left(u_{0}, \ldots, u_{d}\right)$ as above, that

$$
\begin{aligned}
\exp (-\xi) \xi^{d-1} & \leq c_{4} \exp \left\{-\frac{\kappa_{d}}{\tau_{d}}\left(1+c_{1} \varepsilon^{2}\right)\left(1-\frac{c_{1}}{2\left(1+c_{1}\right)} \varepsilon^{2}\right) a \lambda\right\} \\
& \leq c_{4} \exp \left\{-\frac{\kappa_{d}}{\tau_{d}}\left(1+\left(\frac{c_{1}}{2}\right) \varepsilon^{2}\right) a \lambda\right\}
\end{aligned}
$$

Therefore,

$$
\begin{aligned}
\mathbb{P}\left(V_{d}(Z)\right. & \in a[1,1+h], \rho(Z) \geq \varepsilon) \\
& \leq \frac{c_{2}}{d \kappa_{d}^{d-1}} h a \lambda\left(d \kappa_{d}\right)^{d+1} c_{4} \exp \left\{-\frac{\kappa_{d}}{\tau_{d}}\left(1+\left(\frac{c_{1}}{2}\right) \varepsilon^{2}\right) a \lambda\right\} \\
& =c_{5}(d, \varepsilon) h a \lambda \exp \left\{-\frac{\kappa_{d}}{\tau_{d}}\left(1+\left(\frac{c_{1}}{2}\right) \varepsilon^{2}\right) a \lambda\right\},
\end{aligned}
$$

as asserted.

The proof of Theorem 1 is now similar to the final argument in [2]. Let $\varepsilon \in(0,1)$, $a>0$ and $\lambda>0$ with $a \lambda \geq \sigma_{0}>0$ be given, and let $h_{0}$ be as in Lemma 2. Let $I=[a, b)$ be a given interval. The constants $c_{6}, \ldots, c_{9}$ below depend only on $d, \varepsilon$, and $\sigma_{0}$.

If $h_{0}>(b-a) / a$, we put $h_{1}:=(b-a) / a$, then $a\left[1,1+h_{1}\right)=[a, b)=I$. Lemma 2 gives

$\mathbb{P}\left(V_{d}(Z) \in I\right) \geq c_{6}\left(d, \varepsilon, \sigma_{0}\right) h_{1} a \lambda \exp \{-A a \lambda\} \quad$ with $\quad A:=\left(\kappa_{d} / \tau_{d}\right)\left(1+\left(c_{1} / 4\right) \varepsilon^{2}\right)$,

and Lemma 3 gives

$$
\begin{gathered}
\mathbb{P}\left(V_{d}(Z) \in I, \rho(Z) \geq \varepsilon\right) \leq c_{5}(d, \varepsilon) h_{1} a \lambda \exp \{-B a \lambda\} \\
\text { with } \quad B:=\left(\kappa_{d} / \tau_{d}\right)\left(1+\left(c_{1} / 2\right) \varepsilon^{2}\right) .
\end{gathered}
$$

Both estimates together yield

$$
\mathbb{P}\left(\rho(Z) \geq \varepsilon \mid V_{d}(Z) \in I\right) \leq c_{7}\left(d, \varepsilon, \sigma_{0}\right) \exp \{-(B-A) a \lambda\}
$$

with $B-A=\kappa_{d} \tau_{d}^{-1}\left(c_{1} / 4\right) \varepsilon^{2}$.

Suppose now that $h_{0} \leq(b-a) / a$. Then $1+h_{0} \leq b / a$ and $a\left[1,1+h_{0}\right) \subset[a, b)$. Lemma 2 gives

$$
\mathbb{P}\left(V_{d}(Z) \in I\right) \geq c_{6}\left(d, \varepsilon, \sigma_{0}\right) h_{0} a \lambda \exp \{-A a \lambda\}
$$


For $i \in \mathbb{N}_{0}$, Lemma 3 (together with $\left.a\left(1+h_{0}\right)^{i} \lambda \geq \sigma_{0}\right)$ gives

$$
\begin{aligned}
\mathbb{P}\left(V_{d}(Z) \in\right. & \left.a\left(1+h_{0}\right)^{i}\left[1,1+h_{0}\right], \rho(Z) \geq \varepsilon\right) \\
\leq & c_{5}(d, \varepsilon) h_{0}\left(1+h_{0}\right)^{i} a \lambda \exp \left\{-B a\left(1+h_{0}\right)^{i} \lambda\right\} \\
= & c_{5}(d, \varepsilon) h_{0}\left(1+h_{0}\right)^{i} a \lambda \exp \left\{-A a\left(1+h_{0}\right)^{i} \lambda\right\} \exp \left\{-(B-A) a\left(1+h_{0}\right)^{i} \lambda\right\} \\
\leq & c_{5}(d, \varepsilon) h_{0} a \lambda \exp \{-A a \lambda\} \exp \{-((B-A) / 2) a \lambda\} \\
& \times\left(1+h_{0}\right)^{i} \exp \left\{-((B-A) / 2) \sigma_{0}\left(1+h_{0}\right)^{i}\right\} .
\end{aligned}
$$

From $[a, b) \subset \bigcup_{i=0}^{\infty} a\left(1+h_{0}\right)^{i}\left[1,1+h_{0}\right]$ we now get

$$
\begin{aligned}
\mathbb{P}\left(V_{d}(Z) \in\right. & I, \rho(Z) \geq \varepsilon) \\
\leq & c_{5}(d, \varepsilon) h_{0} a \lambda \exp \{-A a \lambda\} \exp \{-((B-A) / 2) a \lambda\} \\
& \times \sum_{i=0}^{\infty}\left(1+h_{0}\right)^{i} \exp \left\{-((B-A) / 2) \sigma_{0}\left(1+h_{0}\right)^{i}\right\} \\
= & c_{8}\left(d, \varepsilon, \sigma_{0}\right) h_{0} a \lambda \exp \{-A a \lambda\} \exp \{-((B-A) / 2) a \lambda\} .
\end{aligned}
$$

Together with (15), this gives

$$
\mathbb{P}\left(\rho(Z) \geq \varepsilon \mid V_{d}(Z) \in I\right) \leq c_{9}\left(d, \varepsilon, \sigma_{0}\right) \exp \{-((B-A) / 2) a \lambda\}
$$

This completes the proof of Theorem 1.

\section{References}

1. A. Goldman, Sur une conjecture de D. G. Kendall concernant la cellule de Crofton du plan et sur sa contrepartie brownienne, Ann. Probab. 26 (1998), 1727-1750.

2. D. Hug, M. Reitzner, and R. Schneider, The limit shape of the zero cell in a stationary Poisson hyperplane tessellation, Ann. Probab. (to appear).

3. I. N. Kovalenko, A proof of a conjecture of David Kendall on the shape of random polygons of large area (Russian), Kibernet. Sistem. Anal. 1997, No. 4, 3-10, 187; Engl. transl. Cybernet. Systems Anal. 33 (1997), 461-467.

4. I. N. Kovalenko, An extension of a conjecture of D. G. Kendall concerning shapes of random polygons to Poisson Voronoï cells, In: Voronoi's Impact on Modern Science, Book I (P. Engel, et al., eds.), Transl. from the Ukrainian. Kyiv: Institute of Mathematics, 276 p. Proc. Inst. Math. Natl. Acad. Sci. Ukr. Math. Appl. 212 (1998), 266-274.

5. I. N. Kovalenko, A simplified proof of a conjecture of D. G. Kendall concerning shapes of random polygons, J. Appl. Math. Stochastic Anal. 12 (1999), 301-310.

6. K. Leichtweiß, Über die affine Exzentrizität konvexer Körper, Arch. Math. 10 (1959), 187-199.

7. J. Matoušek, Lectures on Discrete Geometry, Graduate Texts in Mathematics 212, Springer-Verlag, New York, 2002

8. R. E. Miles, A synopsis of "Poisson flats in Euclidean spaces", Izv. Akad. Nauk Armyan. SSR Mat. 5 (1970), 263-285; reprinted in Stochastic Geometry (E. F. Harding and D. G. Kendall, eds.) Wiley, New York, 1974, pp. 202-227.

9. R. E. Miles, A heuristic proof of a long-standing conjecture of D. G. Kendall concerning the shapes of certain large random polygons, Adv. in Appl. Probab. 27 (1995), 397-417.

10. J. Møller, Random tessellations in $\mathbb{R}^{d}$, Adv. in Appl. Probab. 21 (1989), 3-73. 
11. A. Okabe, B. Boots, K. Sugihara, and S. N. Chiu, Spatial Tessellations; Concepts and Applications of Voronoi Diagrams., 2nd edn., Wiley, Chichester, 2000.

12. R. Schneider, Convex Bodies: the Brunn-Minkowski Theory, Encyclopedia of Mathematics and Its Applications 44, Cambridge University Press, Cambridge, 1993.

13. R. Schneider and W. Weil, Stochastische Geometrie, Teubner Skripten zur Mathematischen Stochastik, Teubner, Stuttgart, 2000.

14. D. Stoyan, W. S. Kendall, and J. Mecke, Stochastic Geometry and Its Applications, 2nd edn., Wiley, Chichester, 1995.

Received November 25, 2002, and in revised form April 15, 2003. Online publication December 19, 2003. 